\title{
Injury Severity and Comorbidities Alone Do Not Predict Futility of Care after Geriatric Trauma
}

\author{
David B. Duvall, MD, ${ }^{1}$ Xiujun Zhu, PhD, ${ }^{2}$ Alan C. Elliott, MAS, MBA, ${ }^{2}$ Steven E. Wolf, MD, ${ }^{3}$ \\ Ramona L. Rhodes, MD, MPH, ${ }^{4}$ M. Elizabeth Paulk, MD, and Herb A. Phelan, MD, MSCS ${ }^{3}$
}

\begin{abstract}
Background: When counseling surrogates of massively injured elderly trauma patients, the prognostic information they desire is rarely evidence based.

Objective: We sought to objectively predict futility of care in the massively injured elderly trauma patient using easily available parameters: age, Injury Severity Score (ISS), and preinjury comorbidities.

Methods: Two cohorts (70-79 years and $\geq 80$ years) were constructed from The National Trauma Data Bank (NTDB) for years 2007-2011. Comorbidities were tabulated for each patient. Mortality rates at every ISS score were tabulated for subjects with 0,1 , or $\geq 2$ comorbidities. Futility was defined a priori as an in-hospital mortality rate of $\geq 95 \%$ in a cell with $\geq 5$ subjects.

Results: A total of 570,442 subjects were identified (age 70-79 years, $n=217,384$; age $\geq 80$ years, $n=352,608$ ). Overall mortality was $5.3 \%$ for ages $70-79$ and $6.6 \%$ for $\geq 80$ years. No individual ISS score was found to have a mortality rate of $\geq 95 \%$ for any number of comorbidities in either age cohort. The highest mortality rate seen in any cell was for an ISS of 66 in the $\geq 80$ year-old cohort with no listed comorbidities (93.3\%). When upper extremes of ISS were aggregated into deciles, mortality for both cohorts across all number of comorbidities was $45.5 \%-60.9 \%$ for ISS $40-49,56.6 \%-81.4 \%$ for ISS $50-59$, and $73.9 \%-93.3 \%$ for ISS $\geq 60$.

Conclusions: ISS and preinjury comorbidities alone cannot be used to predict futility in massively injured elderly trauma patients. Future attempts to predict futility in these age groups may benefit from incorporating measures of physiologic distress.
\end{abstract}

\section{Introduction}

W HEN COUNSELING FAMILIES of the massively injured, the conversation centers around the familial question, "Will my loved one survive?" When having these conversations about elderly trauma patients, it has been our group's anecdotal experience that family members often overestimate the hope of recovery in this cohort. ${ }^{1}$ Physicians have an ethical obligation to the patient and a practical obligation to their medical system and its finite resources to determine when further aggressive efforts at care after traumatic injury are futile.

While few would argue with the importance of the determination of futility after geriatric injury, this decision is usually made subjectively based on the provider's experience rather than being driven by evidence. The paucity of pro- mulgated guidelines for elderly trauma is surprising given the widespread acceptance of an age-incorporated futility model after thermal injury (i.e., the Baux Score ${ }^{2,3}$ ). The need for such a predictive tool after nonthermal injury becomes especially evident when considering that the elderly population is projected to constitute $>20 \%$ of the population by the year $2040 .^{4}$

Previous studies have performed large data bank analysis to predict mortality in the elderly, but have not gained widespread acceptance. ${ }^{5}$ Our goal with this study was to create a simplified model that could reliably predict futility of care in the elderly trauma patient based on assessment of easily obtainable bedside clinical factors, age, preinjury comorbidities, and the patient's Injury Severity Score (ISS). It was our a priori hypothesis that threshold combinations of these scores existed that would correlate with mortality rates

\footnotetext{
${ }^{1}$ Department of Acute Care Surgery, University of Southern California, Los Angeles, California.

${ }^{2}$ Department of Statistical Science, Southern Methodist University, Dallas, Texas.

${ }^{3}$ Division of Burns/Trauma/Critical Care, Department of Surgery, ${ }^{4}$ Division of Geriatrics and Palliative Care, ${ }^{5}$ Division of Palliative Care, UT Southwestern Medical Center, Parkland Memorial Hospital, Dallas, Texas.

Accepted October 31, 2014.
} 
of $80 \%, 95 \%, 99 \%$, and $100 \%$ (in order to accommodate a spectrum of potential definitions of futility of care).

\section{Methods}

This study was an IRB-approved retrospective review utilizing the National Trauma Data Bank (NTDB) from the American College of Surgeons Committee on Trauma, the largest aggregate trauma registry data from accredited trauma centers in the United States. We queried the NTDB between the years 2007 and 2011 for all patients aged 70 or older. The sample was then divided to create two age cohorts: those aged 70 to 79 years, and those aged 80 years and older.

The ISS has been used as a method to quantitatively assign a score to the magnitude of injury since its description in the 1970 s. $^{6}$ A score of 0 (uninjured) to 5 (massively injured) is assigned to six different body areas, and the ISS is obtained by squaring all injured areas and summing the squares of the three worst scores. ISS scores can therefore range from 0 for an uninjured person to a maximum of $75\left(5^{2}+5^{2}+5^{2}=75\right)$. This data is ordinal and the range of scores are not consecutive (i.e., the ISS that is the next highest to the maximum of 75 is the sum of $5^{2}+5^{2}+4^{2}$, or 66). Finally, an important exception to this general rule of ISS calculation lies in a set of predefined injuries which are considered untreatable (i.e., decapitation), and these injuries have a body area score of 6 and an automatic overall ISS of 75 regardless of what other body areas may have been injured. Due to the fact that family discussions for these patients with untreatable injuries are moot, we chose to exclude all subjects with an ISS score of 75 from our analysis. We then began by calculating overall mortality for each individual ISS score without consideration of comorbidities.

The NTDB during the years in question tracked 27 different preinjury comorbidities. Two were excluded due to nonapplicability to a geriatric cohort (prematurity and congenital anomalies), and two due to their lesser impact on outcomes (hypertension requiring medication and smoking). "Presence of an advanced directive limiting care" was excluded due to its potential confounding effect by causing death despite a medically survivable injury. This left 22 comorbidities for inclusion in the analysis (see Table 1). The two age cohorts were then further subdivided by grouping the patients as having 0,1 , or $\geq 2$ of these comorbid conditions.

All subgroups were then stratified according to their ISS by deciles, and mortality rates were calculated during the index hospital stay by number of comorbidities for each decile. As we were searching for signals of futility, we restricted our comorbidity analyses to massively injured subjects, defined here with an ISS of $\geq 40$. Futility was defined a priori as an inhospital mortality rate of $\geq 95 \%$ for any cell containing at least five patients. All analyses were performed using statistical software SAS (SAS version 9.3; SAS Institute Inc., Cary, NC).

\section{Results}

The query resulted in 570,422 subjects that met inclusion criteria. Of these, 217,384 subjects were ages 70 to 79 , while 352,608 subjects were ages 80 or older. The overall mortality rates for the two cohorts were $5.3 \%$ and $6.6 \%$, respectively.

When examining the mortality rate by individual ISS score, no score surpassed the a priori requirement of a $95 \%$ mortality even at the extremes of ISS. In the 70 to 79 year-old cohort, the highest mortality rate seen with any one score was
Table 1. National Trauma Data Bank COMORBIDITIES INCLUdED IN THE ANALYSIS

NTDB Comorbidities included in the analysis

Alcoholism

Ascites within 30 days

Bleeding disorder

Chronic renal failure

Cirrhosis

Currently receiving chemotherapy

Congestive heart failure

CVA/residual neurologic deficit

Dementia

Diabetes mellitus

Disseminated cancer

Drug abuse or dependence

Esophageal varices

Functionally dependent health status

History of angina within last 30 days

History of myocardial infarction

History of peripheral vascular disease

Major psychiatric illness

Obesity

Prehospital cardiac arrest with CPR

Respiratory disease

Steroid use

for an ISS score of 59, which had an overall associated death rate of $74.7 \%$ (see Fig. 1). In the 80 years or older cohort, the highest mortality rate was seen with an ISS score of 66 $(89.5 \%$ ) (see Fig. 2). Of those ISS scores excluded from the comorbidity analyses by virtue of their being unlikely to approach futility, the highest mortality rates were $45.7 \%$ for the 70 to 79 year-old sample (an ISS score of 38), and $61.6 \%$ for the 80 years or older sample (also an ISS of 38).

After restricting the analysis to subjects with an ISS of $\geq 40$, the 70 to 79 year-old sample had 1511 subjects remaining while the $\geq 80$ year old sample had 1115 (see Table 2 ). For subjects ages 70 to 79 years with two or more comorbidities, the mortality rate was $46.8 \%$ if they sustained an ISS in the 40s and $56.6 \%$ if they had an ISS in the 50s. Thirty subjects in this cohort had an ISS score of 66 (i.e., the only ISS score possible in the 60s). Of these, only three had a single comorbidity listed, while four had two or more comorbidities listed; therefore mortality rates were not calculated for these cells. Of the 23 patients ages 70 to 79 years who had no comorbidities listed and an ISS score in the 60s, the mortality rate was $73.9 \%$.

For subjects ages $\geq 80$ years with two or more comorbidities, the mortality rate was $60.9 \%$ if they sustained an ISS in the $40 \mathrm{~s}$ and $81.4 \%$ if they had an ISS score in the $50 \mathrm{~s}$. Nineteen subjects in this cohort had an ISS score of 66. Of these, only four had any comorbidities listed at all, and therefore mortality rates were not calculated for these cells. Of the 15 patients ages 80 years or older who had no comorbidities listed and an ISS score in the 60s, the mortality rate was $93.3 \%$.

\section{Discussion}

Counseling families of severely injured geriatric trauma patients is problematic for a variety of reasons. The conversation tends to be laden with terms like "increased 


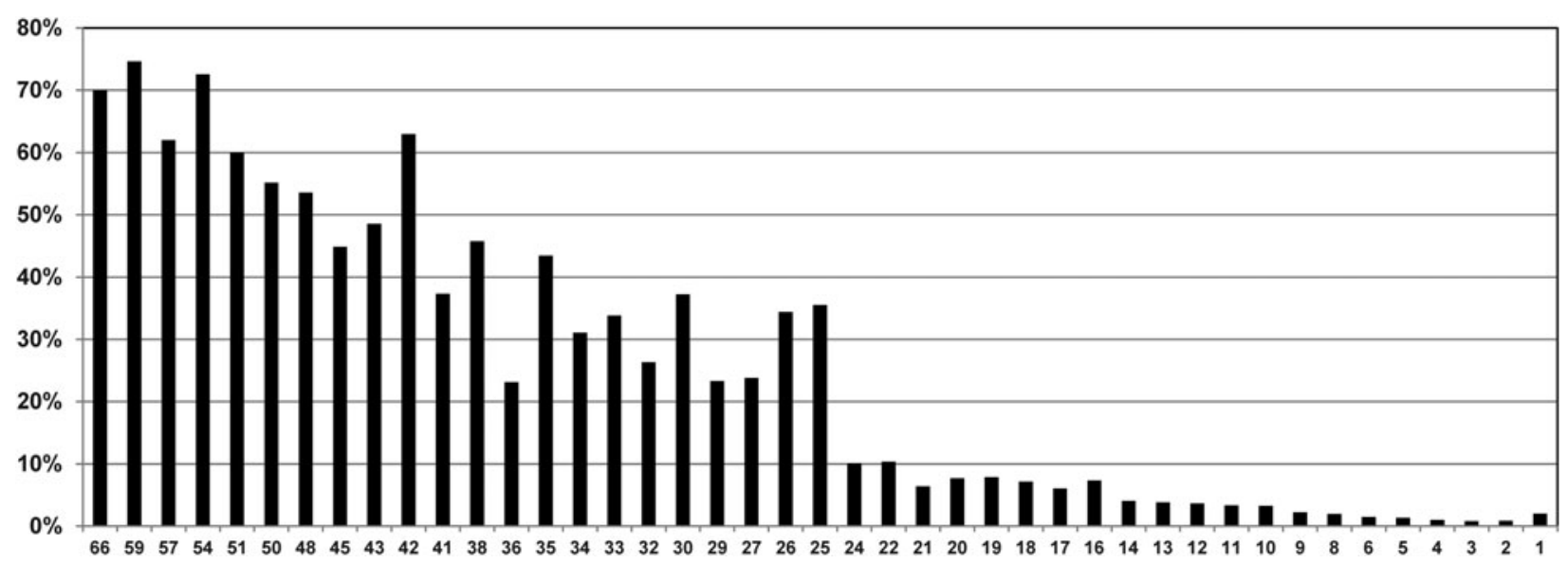

FIG. 1. Mortality rates for all possible Injury Severity Scores regardless of comorbidities in the 70 to 79 year-old sample.

probability," "often," "usually," "more likely than not," etc. We have found that the subjectivity of these words and phrases can be frustrating for families when having to make decisions about the aggressiveness of care in this population when a bad outcome appears likely but not certain. In turn, it is common for families experiencing frustration at the subjectivity of these terms to default to an aggressive treatment strategy. Conversely, in our groups' experiences, families sometimes experience relief when told that the severity of injury has rendered further care futile with a strong recommendation for the initiation of comfort care. This recommendation can help alleviate feelings of guilt on the part of legally authorized representatives who might otherwise feel an element of responsibility for the patient's death.

The objective determination of futile care in geriatric trauma patients has lagged behind the efforts towards this same goal after thermal injury. Burn surgeons have utilized the Baux Score since its description in 1961 for this means. ${ }^{2}$ In its original iteration, the Baux Score used the sum of two easily derived variables (age and percent total body surface area burned) to identify futility of care, with a sum of 140 generally agreed upon as a lethal burn. In 2010, advanced statistical modeling was used to update the Baux Score based on a large sample from the National Burn Repository by taking into account the effects of inhalation injury as well as modern burn care. ${ }^{3}$ By adding a score of 17 to the original Baux Score calculation if an inhalation injury was present, a nomogram was generated that indicated scores associated with a $95 \%$ probability of death (a revised Baux Score of 150), 99\% (revised Baux Score of 173), and $100 \%$ (score of 200). The advantages of this scoring system are ease of calculation as well as comprehensibility to a layman.

In the interest of simplicity, we sought to restrict the variables to those that would be easily identifiable as well as available at the time of a family meeting. This led us to select age, ISS, and preinjury comorbidities as our variables of interest. While this strategy is inoperative in the emergency department, in our groups' practices a determination of futility only tends to occur in the emergency department in the context of a devastating head injury as diagnosed by our neurosurgery services. Barring a diagnosis of nonsurvivability by our neurosurgeons, we tend to be aggressive

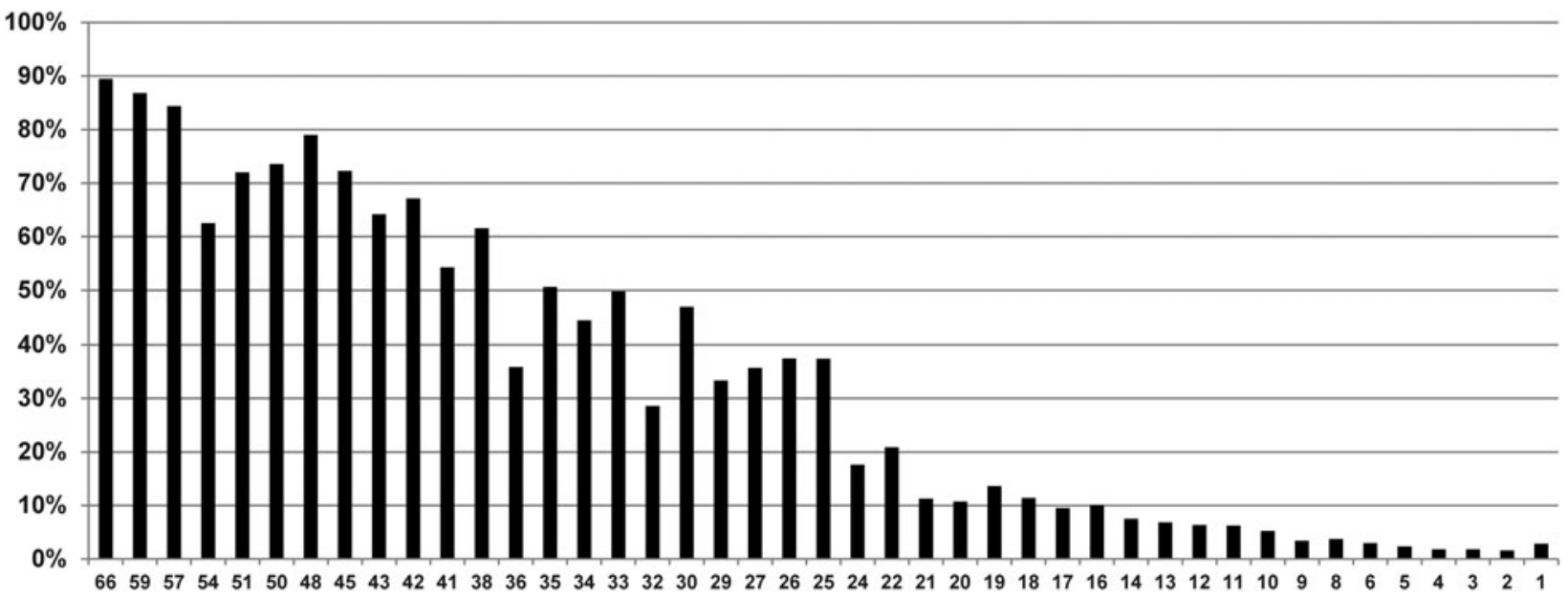

FIG. 2. Mortality rates for all possible Injury Severity Scores regardless of comorbidities in the 80 years and older sample. 
Table 2. Mortality Rates for Each Age Cohort Grouped by Number of Comorbidities and InJury Severity Score Deciles

\begin{tabular}{|c|c|c|c|c|c|c|c|c|c|}
\hline \multirow[b]{2}{*}{ Number of comorbidities } & \multicolumn{3}{|c|}{ ISS $40-49$} & \multicolumn{3}{|c|}{ ISS 50-59 } & \multicolumn{3}{|c|}{$I S S \geq 60$} \\
\hline & 0 & 1 & $\geq 2$ & 0 & 1 & $\geq 2$ & 0 & 1 & $\geq 2$ \\
\hline \multicolumn{10}{|l|}{ Age $70-79$ yrs } \\
\hline Deaths/subjects & $301 / 661$ & $104 / 244$ & $44 / 94$ & $203 / 326$ & $60 / 103$ & $30 / 53$ & $17 / 23$ & $1 / 3$ & \\
\hline Mortality rate & $45.5 \%$ & $42.6 \%$ & $46.8 \%$ & $62.3 \%$ & $58.3 \%$ & $56.6 \%$ & $73.9 \%$ & NA & NA \\
\hline \multicolumn{10}{|l|}{ Age $\geq 80$ yrs } \\
\hline Deaths/subjects & $291 / 449$ & $126 / 209$ & $56 / 92$ & $175 / 230$ & $64 / 89$ & $22 / 27$ & $14 / 15$ & $2 / 3$ & $1 / 1$ \\
\hline Mortality rate & $64.8 \%$ & $60.3 \%$ & $60.9 \%$ & $76.1 \%$ & $71.9 \%$ & $81.4 \%$ & $93.3 \%$ & NA & NA \\
\hline
\end{tabular}

ISS, Injury Severity Scores.

with our care until hemorrhage control has been achieved (if needed) and/or the patient arrives in the intensive care unit. It was our hope that we would be able to demonstrate scenarios that corresponded to universal lethality (i.e., a 100\% probability of dying and therefore no quibbling about the definition of "futility"), as well as $99 \%$ and $95 \%$ probabilities, which laymen could find instructive if not definitive.

We were surprised to find that even at exceedingly high ISS scores, no combination of ISS, age, and comorbidity demonstrated a $95 \%$ death rate among these geriatric cohorts. Several explanations are possibilities for the interpretation of these results.

It is possible that these results represent a true signal. As improvements in trauma care have proceeded in the realms of prehospital transport, resuscitative strategies, and critical care techniques, it is possible that the tolerance for injury in this geriatric population is actually much higher than would appear at first glance. It is clear that this population's decreased physiologic reserves put them at higher risk for a bad outcome injury for injury than their younger counterparts, but a determination of futility as defined here may not be possible on arrival to the SICU.

Another possibility is that the determination of futility in the elderly will require some measure of physiologic distress. The most clinically intuitive surrogate for physiologic distress is the number of units of packed red blood cells (PRBCs) transfused during the initial resuscitation. The NTDB does not currently capture this variable, however. A surrogate captured by the NTDB that may have predictive utility is the measurement of the base deficit at the time of arrival in the emergency department. This is a missing data point for many patients, however, and furthermore it is not missing at random, as its measurement is frequently omitted when patients arrive in the emergency department in an unstable condition. We felt that these confounders precluded its use in our model.

Parenthetically, our group is currently addressing this limitation in a follow-up study conducted with our own trauma databank in which these variables are available. The easiest marker to assess at the bedside is the volume of PRBC transfusion that has occurred at the completion of resuscitation. Our local trauma data bank tracks this information as well as adheres strictly to the a priori definitions for "lethal" injuries (and therefore an automatic designation of an ISS of 75) promulgated by the American College of Surgeons. It should be stressed that one of the primary features of this scoring system when created has to be simplicity. A scoring system for mortality and futility based on age, ISS, and amount of blood transfused is one that any trauma surgeon would be able to calculate with ease.

An additional possibility for our inability to find a $95 \%$ mortality rate for even the highest severities of injury may lie in our decision to exclude those subjects with ISS scores of 75. As stated previously, the list of injuries designated "untreatable" and therefore mandating an ISS score of 75 have been defined a priori by the American College of Surgeons. A part of all trauma centers' quality improvement (QI) processes is for all deaths to be adjudicated as "nonpreventable," "nonpreventable with opportunities for improvement," and "preventable." In conversations with medical coders and registrars (who are the personnel who physically input trauma data including ISS scores and death adjudications into institutional registries which form the basis of the NTDB), it is a common error for inexperienced coders to consider a determination of a nonpreventable death as "untreatable" and enter it as an ISS of 75.

We will use Parkland's most famous death as an example of how this error can impact a study such as ours. John F. Kennedy sustained one gunshot wound to the head and one to the upper back which exited his throat. The head and neck are considered to be a contiguous unit for the purposes of ISS calculation and would have merited a score of 5 . The wound to his upper back is counted as a chest wound and would merit a score of 4 . Therefore, his ISS score would be $5^{2}+4^{2}=41$, and his death is widely known to be "nonpreventable." Were a medical coder to incorrectly assign him an ISS score of 75 based on nonpreventability, he would then have been excluded from an analysis such as ours. It is apparent, then, that this practice would systematically produce a survival bias for those patients with ISS scores other than 75 .

The development and promulgation of a widely accepted tool for the prediction of futility has been elusive. Boyd described the Trauma Score-Injury Severity Score (TRISS) in 1987 as a method of calculating odds of death after traumatic injury, which was centered around age, ISS, mechanism of injury (blunt or penetrating), prehospital respiratory rate, Glasgow Coma Score, and systolic blood pressure. ${ }^{7}$ The confounding effect of intubation on its calculation and the fact that its dataset reflects the standard of trauma care as it was delivered almost 30 years ago prevents its widespread use. In 2003, Nirula used an analysis of the NTDB from the years 1994 to 2001 and found that in trauma patients age 65 years or older with various combinations of head, chest, and abdominal injuries in the setting of a presenting systolic blood pressure of $90 \mathrm{mmHg}$ and a base deficit of -12 , 
mortality rates greater than $95 \%{ }^{5}$ could be found. It is possible that study's omission of ISS scores and reliance on base deficit has handicapped its widespread adoption.

In 2011 Barbosa and colleagues examined a sample of 704 subjects of all ages who had received a massive transfusion of blood (conventionally defined as a transfusion of 10 units of PRBCs or more in the first 24 hours after injury) $)^{8}$ in an unsuccessful attempt to find physiologic indicators of futility regardless of age. Amongst their findings, they noted that all 15 subjects age 65 years and older who had a head injury scored as a 5 by the ISS schema and who were massively transfused, died.

It should be mentioned that all of these studies (present one included) focus solely on death during index admission as the endpoint for defining futility of care in the elderly after trauma. Unfortunately, it has been our group's experience that families frequently hold a naive belief that survival, should it occur, will mean a return to the previous functional baseline for the injured elderly. Future work in this field is going to require being able to prognosticate about life and limitations after discharge for those who survive given that accurate assessments of changes in functional status will assuredly factor into the decision making of legally authorized representatives.

At present, our multidisciplinary group is applying for extramural funding to address these questions. It is our plan to prospectively follow a large cohort of geriatric trauma patients from eight North American trauma centers for six months after injury. In so doing, we will be able to gain insight into functional status as well as delayed mortality after serious injury. We plan to track quality of life scores, pain management, symptom burden, and Katz scores for activities of daily living. Additionally, we will track hospice and emergency department utilization, subsequent hospitalizations, and 30-day readmission rates after discharge. We also plan to track the impact of the convalescence on caregivers as well as family satisfaction with the decisions that they made. We are calling it the "Prospective $\boldsymbol{A}$ ssessment of $\boldsymbol{L}$ ife and $\boldsymbol{L} \boldsymbol{I m i t a t i o n s} \boldsymbol{A}$ fter $\boldsymbol{T}$ rauma in the $\boldsymbol{E} \overline{\text { lderly" (PAL- }}$ LIATE) study, and we have high hopes that it will significantly advance the cause of providing optimal care for the severely injured geriatric trauma patient.

In summary, patient age, injury severity score, and comorbidities are not able to be used to predict futility of care (defined as a 95\% probability of dying) for the elderly after injury. Future efforts to create a model may benefit from utilizing a consistently captured marker of physiologic distress. An obligation exists to patients and society to develop and promulgate objective identifiers of futility.

\section{Acknowledgments}

The National Trauma Data Bank is the property of the Committee on Trauma, American College of Surgeons. The content reproduced from the NTDB remains the full and exclusive copyrighted property of the American College of Surgeons. The American College of Surgeons is not responsible for any claims arising from its data.

\section{Author Disclosure Statement}

No competing financial interests exist.

\section{References}

1. Richmond TS, Kauder D, Strumpf N, et al.: Characteristics and outcomes of serious traumatic injury in older adults. J Am Geriatr Soc 2002;50:215-222.

2. Baux S: Contribution a l'etude du traitement local des brulures thermiques etendues. Thesis. Paris, 1961.

3. Roberts G, Lloyd M, Parker M, et al.: The Baux score is dead. Long live the Baux score: A 27-year retrospective cohort study of mortality at a regional burns service. J Trauma Acute Care Sur 2012;72:251-256.

4. Pellicane JV, Byrne K, DeMaria EJ: Preventable complications and death from multiple organ failure among geriatric trauma victims. J Trauma 1992;33:440-444.

5. Nirula R, Gentilello LM: Futility of resuscitation criteria for the "young" old and the "old" old trauma patient: A National Trauma Data Bank analysis. J Trauma 2004;57:37-41.

6. Baker SP, O'Neill B, Haddon Jr W, et al.: The Injury Severity Score: A method for describing patients with multiple injuries and evaluating emergency care. J Trauma 1974;14: 187-196.

7. Boyd CR, Tolson MA, Copes WS: Evaluating trauma care: The TRISS method. J Trauma 1987;27:370-378.

8. Barbosa RR, Rowell SE, Diggs BS, et al.: Profoundly abnormal initial physiologic and biochemical data cannot be used to determine futility in massively transfused trauma patients. J Trauma 2011;71:S364-S369.

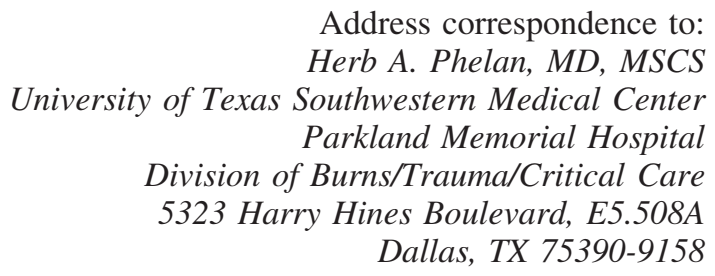

E-mail: herb.phelan@utsouthwestern.edu 